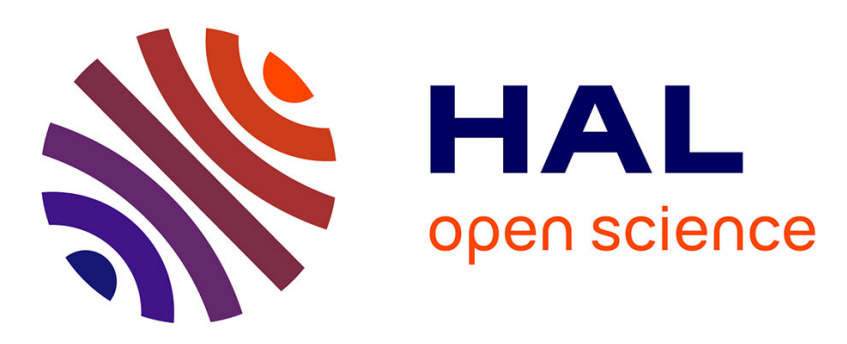

\title{
Resolving the root of the avian mitogenomic tree by breaking up long branches.
}

Kerryn E Slack, Frédéric Delsuc, Patricia A Mclenachan, Ulfur Arnason, David Penny

\section{- To cite this version:}

Kerryn E Slack, Frédéric Delsuc, Patricia A Mclenachan, Ulfur Arnason, David Penny. Resolving the root of the avian mitogenomic tree by breaking up long branches.. Molecular Phylogenetics and Evolution, 2007, 42 (1), pp.1-13. 10.1016/j.ympev.2006.06.002 . halsde-00315505

\section{HAL Id: halsde-00315505 https://hal.science/halsde-00315505}

Submitted on 28 Aug 2008

HAL is a multi-disciplinary open access archive for the deposit and dissemination of scientific research documents, whether they are published or not. The documents may come from teaching and research institutions in France or abroad, or from public or private research centers.
L'archive ouverte pluridisciplinaire HAL, est destinée au dépôt et à la diffusion de documents scientifiques de niveau recherche, publiés ou non, émanant des établissements d'enseignement et de recherche français ou étrangers, des laboratoires publics ou privés. 
Resolving the root of the avian mitogenomic tree by breaking up long branches.

\author{
Kerryn E. Slack ${ }^{1,2}$, Frédéric Delsuc ${ }^{1,3}$, P.A. (Trish) McLenachan ${ }^{1}$, \\ Ulfur Arnason $^{2}$ and David Penny ${ }^{1}$
}

\author{
${ }^{1}$ Allan Wilson Center for Molecular Ecology and Evolution, \\ Massey University \\ Palmerston North \\ New Zealand \\ ${ }^{2}$ Department of Genetics, Cell and Organism Biology \\ University of Lund \\ Lund \\ Sweden \\ ${ }^{3}$ Present address: \\ Laboratoire de Paléontologie, Phylogénie et Paléobiologie, \\ Institut des Sciences de l'Evolution UMR5554-CNRS \\ Université Montpellier II \\ Montpellier \\ France
}

Keywords: avian evolution, long branches, galloanseres, Passeriformes, Neoaves, Cracrafti 


\begin{abstract}
:
Incomplete taxon sampling has been a major problem in resolving the early divergences in birds. Five new mitochondrial genomes are reported here and three break up long branches that tended to attract the distant reptilian outgroup. These long branches were to galliforms, and to oscine and suboscine passeriformes.

Breaking these long branches leaves the root between paleognaths and neognaths as inferred by maximum likelihood and Bayesian phylogenetic analyses. This means that morphological, nuclear and mitochondrial data are now in agreement on the position of the root of the avian tree and we can, for the moment, move on to other questions. An overview is then given of the deepest divisions in the mitogenomic tree inferred from complete mitochondrial genomes. The strict monophyly of both the galloanseres and the passerines is strongly supported, leaving the deep six-way split within Neoaves as the next major question for which resolution is still lacking. Incomplete taxon-sampling was a major problem earlier and although some resolution is now available there are still problems related to the fact that current phylogenetic methods still fail to account for real features of DNA sequence evolution.
\end{abstract}




\section{Introduction}

After a period of relative quiescence following the publication of Sibley and Ahlquist's DNA/DNA hybridisation tapestry (1990) the ever-decreasing cost of DNA sequences has led to a rebirth of deep-level avian systematics. As a part of this effort to acquire new data aiming at resolving the phylogeny of avian orders, complete mitochondrial genomes had been sequenced for an increasing number of taxa (for example, Mindell et al. 1999; Paton et al. 2002; Slack et al. 2003; Harrison et al. 2004). The amount of sequence data accumulated so far permits the evaluation of a range of prior hypotheses primarily proposed on morphological, paleontological and biogeographical grounds (for example, Cracraft 2001; Cracraft et al. 2004). In the case of placental mammals, as expected on theoretical grounds, increasing the number of taxa led to an excellent agreement between nuclear and mitochondrial datasets (Lin et al. 2002; Reyes et al. 2004). Such agreement between different datasets is essential for corroboration (Penny et al. 1982) because model misspecification (Buckley 2002) can be hard to detect, Systematic biases can lead to 100\% bootstrap support for conflicting trees (Phillips et al. 2004), and even maximum likelihood methods can become inconsistent when the model is not specified accurately (Chang 1996).

An early problem was that mitogenomic-based studies of avian phylogenetic relationships appeared to place the reptilian outgroup within Passeriformes (Härlid and Arnason 1999; Mindell et al. 1999). Such a rooting was at odds with data from morphology (Sibley and Ahlquist 1990; Cracraft 2001; Livezey and Zusi 2001; Mayr and Clarke 2003), nuclear DNA (Garcia-Moreno and Mindell 2000) and genomic DNA strings (Edwards et al. 2002). These all supported a major division between paleognaths (tinamous and ratites) and neognaths (all other birds including the species-rich Passeriformes). The passerine rooting of the mitochondrial tree has been suggested to be the result of a possible long-branch attraction effect between the distant reptilian outgroup and the fast-evolving passerines (Slack et al. 2003). Indeed, in the unrooted avian tree the passerines were grouped together on the tree, but could become paraphyletic, or even diphyletic when the outgroup was added (Slack et al. 2003). Such disruption of the ingroup by a distant outgroup is known from simulation studies (Holland et al. 2003) and here the ingroup tree by itself was more likely to be correct. A similar ingroup disruption was also found with mammals (Lin et al. 2002). In general, distant outgroups are hard to place correctly into the ingroup tree (Holland et al. 2003). Taxon sampling from Passeriformes has been sparse with only one suboscine (broadbill) and two oscines (rook and indigobird) included at that time (Braun and Kimball 2002). Base composition bias might also have played a role since phylogenetic reconstructions under a non-homogeneous model dealing with base composition heterogeneity (Paton et al. 2002) and RY-coding analyses (Braun and Kimball 2002) supported the classical rooting of the avian tree between paleognaths and neognaths. However, the position of the root of the avian tree is still difficult to resolve clearly with complete mitochondrial data and must be 
tested by the inclusion of more basal neognaths (Galloanserae) as well as Passeriformes that appear especially fast evolving (Harrison et al. 2004). Ideally, it is preferable for a result to be found by all good methods of phylogenetic analysis, not just by one specialised analysis. In difficult cases, it might thus be necessary for both theoretical and practical reasons to first build an unrooted tree, and then test the placement of the root secondarily.

The phylogeny of paleognaths, with a particular emphasis on both living and extinct ratites, has been well studied from the mitogenomic viewpoint (Härlid and Arnason 1999; Härlid et al. 1999; Cooper et al. 2001; Haddrath and Baker 2001). Their origins and biogeographic evolution in relation to the tectonic fragmentation of Gondwana are now relatively well understood (Cooper et al. 2001; Haddrath and Baker, 2001), although some relationships within ratites and between deep ratites and tinamous are still uncertain. Turning to neognaths, they are usually divided into Galloanserae (chicken, ducks and allies) and Neoaves (all other neognath birds). This subdivision is now well supported from molecular data by both mitochondrial (Paton et al. 2002; Harrison et al. 2004) and nuclear sequences (van Tuinen et al. 2000; Garcia-Moreno et al. 2003; Chubb 2004). We have recently added the magpie goose (Anseranas semipalmata) a basal member of the water fowl lineage (Anseriformes) to the complete mitochondrial dataset, establishing an important calibration point for avian evolution (Harrison et al. 2004). In the present study, we include a basal member (Ericson et al. 2002b) of the chicken-related lineage (Galliformes) by sequencing the complete mitochondrial genome of the Australian brush-turkey (Alectura lathami, Megapodiidae). This is expected to break the relatively long ancestral lineage leading to chicken and quail, and to test the relationships within Galloanserae further.

Phylogenetic relationships among the six or more major groups of Neoaves that contain the vast majority of extant bird species remain elusive and are usually represented as a multifurcation (Cracraft 2001; Cracraft et al. 2004). Within this unresolved phylogeny, Passeriformes are by far the most speciose group. The current taxonomy of oscines, based mainly on the results of DNA/DNA hybridizations (Sibley and Ahlquist 1990), has recently been challenged by analyses of both nuclear and mitochondrial genes (Irestedt et al. 2001; Ericson et al. 2002a,b; Ericson and Johansson 2003; Barker et al. 2004). Based on these new results a new taxonomy of major passerine groups has been proposed (Ericson et al. 2002b). Three major lineages have been distinguished: Acanthisittia (New Zealand wrens), suboscines (Eurylaimides or Old World suboscines and Tyrannides or New World suboscines) and oscines (Menurae and Euoscines). Oscines and Suboscines are grouped together into Eupasseres to the exclusion of New Zealand wrens, the latter representing the most basal lineage of Passeriformes. The early emergence of New Zealand wrens 
within Passeriformes is consistent with a Gondwanan origin for the whole passerine group (Cracraft 2001; Barker et al. 2002; Edwards and Boles, 2002; Ericson et al. 2002a).

The passerines have been represented in the mitogenomic database by only three taxa: the gray-headed broadbill (Smithornis sharpei; Eurylemidae) belonging to the Old World suboscines, and two Euoscines: the village indigobird (Vidua chalybeata; Estrildidae) and the rook (Corvus frugilegus; Corvidae). Under the new classification (Ericson et al. 2002b) rook and indigo bird are both in the Euoscines, leaving the Menurae unrepresented. A representative of New Zealand wrens, the rifleman (Acanthisitta chloris; Acanthisittidae) has been recently added to the complete mitochondrial dataset, but its position was still locally unstable within passerines (Harrison et al. 2004). By incorporating the new complete mitochondrial genomes of the superb lyrebird (Menura novaehollandiae; Menuridae) representing the second major group of oscines, and the fuscous flycatcher (Cnemotriccus fuscatus; Tyrannidae) a member of the New World suboscines, we now have a much more representative taxon sampling of Passeriformes. We expect from theory (Hendy and Penny 1989) and from simulations (Holland et al. 2003) that these key taxa will stabilise the relationships within Passeriformes by subdividing the two long branches leading to oscines and suboscines, respectively. This is especially important in order to tackle issues such as the position of the rifleman within passerines, and especially the central question of the position of the root of the avian tree.

Another interesting aspect of the current mitochondrial tree is the difficulty (Haring et al. 2001; Slack et al. 2003; Harrison et al. 2004) in recovering the expected monophyly of birds of prey (Falconiformes) currently represented by the buzzard (Buteo buteo; Accipitridae) and the falcon (Falco peregrinus; Falconidae). Falconiformes are part of a large group of Neoaves including seabirds, shorebirds, doves, cranes, rails, flamingos, penguins, loons and grebes (Cracraft, 2001), a major grouping we informally call the Conglomerati (or Cracrafti). The buzzard and falcon share the same alternative mitochondrial gene order, but are fairly divergent from each other and seem to represent an early split in the raptor lineage (Haring et al. 2001). Thus we again have sparse taxon sampling. Previously, only partitioned-likelihood phylogenetic analyses (with RY-coding third codon positions of the mitochondrial proteins plus nucleotides from RNAs) appearing to support their monophyletic origin (Harrison et al. 2004).

As a first step we have sequenced the complete mitochondrial genome of the turkey vulture (Cathartes aura; Cathartinae). This provides an opportunity to test the Sibley and Ahlquist hypothesis (1990) that New World vultures (Cathartinae) are closer to storks (Ciconiinae) than to other birds of prey. It has long being realised that New World and Old World vultures may not share a most recent common ancestor, but may instead represent an example of morphological convergence resulting from adaptation to a scavenging way 
of life (see Sibley and Ahlquist 1990 for an historical review). However, the question of whether New World vultures are more closely related to storks, as suggested by behavioural resemblances (Rea, 1983) and DNA/DNA hybridisations (Sibley and Ahlquist 1990), or to birds of prey (including Old World vultures) is still ambiguous (Seibold and Helbig 1995). Resolving this question certainly requires more molecular data (Helbig and Seibold 1996).

The order Charadriiformes represents a very large and diversified group of shorebirds which also belongs to the Conglomerati/Cracrafti (Cracraft 2001). Shorebirds are usually divided in three major clades: Charadrii (oystercatchers, thick-knees, sheathbills, plovers, and allies), Scolopaci (turnstones, sandpipers and jacanas), and Lari (gulls, coursers, pratincoles, terns, skimmers, and skuas) (see Van Tuinen et al. 2004). DNA/DNA hybridisation suggested an early emergence of Scolopaci with a sister-group relationship between Charadrii and Lari (Sibley and Ahlquist 1990). The first two shorebird mitochondrial genomes to be sequenced were the blackish oystercatcher (Haematopus ater; Haematopodidae) and the ruddy turnstone (Arenaria interpres; Scolopacidae) (Paton et al. 2002). Since then, data from nuclear genes have explored the relationships among shorebirds families (Ericson et al. 2003; Paton et al. 2003; Thomas et al. 2004). These have challenged the DNA/DNA hybridisation results by finding a closer relationship between Scolopaci and Lari with an early divergence of Charadrii. The sequencing of the southern blackbacked gull (Larus dominicanus; Laridae) mitochondrial genome offers the opportunity to test the new nuclear-based hypothesis by adding a representative of the previously unsampled third major group of Charadriiformes (Lari, gulls).

Here we report mitochondrial genomes for five birds chosen to help clarify the deepest divisions and test specific phylogenetic hypotheses in the avian tree. The sequences have been determined for the brush turkey (Alectura lathami, Megapodiidae), two passerines with a flycatcher (Cnemotriccus fuscatus; Tyrannidae) and the superb lyrebird (Menura novaehollandiae; Menuridae), the turkey vulture (Cathartes aura; Cathartinae) and a gull (Larus dominicanus; Laridae). The phylogenetic analyses using maximum likelihood and Bayesian methods support a growing consensus from nuclear, mitochondrial and morphological data for the position of the root of the avian tree and for its first main divisions. The turkey vulture is not positioned with the stork and the gull joins with the turnstone.

\section{Materials and Methods}

\section{Tissue samples}

The southern black-backed gull (Larus dominicanus [Lichtenstein, 1823]) came from Waikanae, New Zealand and was supplied by the New Zealand Department of Conservation (Kapiti Area branch). The 
Australian brush-turkey (Alectura lathami [Gray, 1831]) and superb lyrebird (Menura novaehollandiae [Latham, 1802]) samples came from Australia. Darryl Jones (Australian School of Environmental Studies, Griffith University, Brisbane) and Ian Owens (Queensland University) provided the brush-turkey, and Cathy Nock (Centre for Animal Conservation Genetics, Southern Cross University [Lismore campus], New South Wales) the lyrebird. The turkey vulture (Cathartes aura [Linnaeus, 1758]) came from Texas, USA and the fuscous flycatcher (Cnemotriccus fuscatus [Wied-Neuwied, 1831]) from Peru. Both samples were supplied by the Louisiana State University Museum of Natural Science Collection of Genetic Resources (sample numbers: turkey vulture LSUMNS B-17242, flycatcher LSUMNS B-7284).

\section{DNA extraction and sequencing}

Genomic DNA was extracted from 25-50 mg of sample tissue using the High Pure ${ }^{\mathrm{TM}}$ PCR Template Preparation Kit (Protocol Vb; Boehringer Mannheim). To minimize the possibility of obtaining nuclear copies of mitochondrial ( $\mathrm{mt}$ ) genes (numts), the five mitochondrial genomes were amplified in two (turkey vulture, flycatcher) or three (gull, brush-turkey, lyrebird) overlapping fragments using long range PCR. The long range products (see below) were recovered from agarose gels using an appropriate kit (e.g. Concert ${ }^{\mathrm{TM}}$ Rapid Gel Extraction System kit [GIBCO BRL/Life Technologies], AccuPrep ${ }^{\mathrm{TM}}$ Gel Purification Kit [Bioneer]). They were then used as templates in a second round of PCR amplification (overlapping fragments of $0.3-3.1 \mathrm{~kb}$ in length). PCR reactions were set up using the long range and reamplification conditions described in Slack et al. (2003). Annealing temperatures for the reamplifications ranged from 48 to $60^{\circ} \mathrm{C}$ and extension times from 1 to 3 minutes. The conserved primers used were from Kocher $e t a l$. (1989), Sorenson et al. (1999), Cooper et al. (2001) and Cooper et al. (unpublished), with some being modified from the original, or were designed from alignments of bird mitochondrial genes. Where necessary (e.g. control region), specific primers were designed for individual birds using the Oligo® 4.03 program (National Biosciences, Inc.).

The positions and sizes of the long-range PCR products and the annealing temperatures, extension times and primers used to generate them are given below. Primer nomenclature: Av (avian) position gene direction (F: forward; R: reverse). Positions and genes indicate where the 3' end of the primer binds in the chicken mitochondrial genome. To avoid confusion, 'gene' and 'direction' are inverted for rRNA primers.

\section{$12 \mathrm{~S}$ or $16 \mathrm{~S}$ rRNA to tRNA-Leu(CUN):}

Turkey vulture and flycatcher $(11.3 \mathrm{~kb}): 60^{\circ} \mathrm{C}, 8 \mathrm{~min}$ Gull, brush-turkey and lyrebird $(9.3 \mathrm{~kb}): 53^{\circ} \mathrm{C}, 9.5 \mathrm{~min}$ and $2 \times 54^{\circ} \mathrm{C}, 10 \mathrm{~min}$, respectively Turkey vulture and flycatcher: Av1753F12S (25 nt): 5'-AAACTGGGATTAGATACCCCACTAT-3' 
Gull, brush-turkey and lyrebird: Av3725F16S-LR (32 nt): 5'-

\section{AATAGGGTTTACGACCTCGATGTTGGATCAGG-3'}

All five birds: Av13026tLeuR2-LR (37 nt): 5'-

\section{CTTGGAKTTGCACCAAGRTDVTTGGTTCCTAAGACCA-3'}

COIII to tRNA-Pro:

Gull and brush-turkey $(5.5 \mathrm{~kb}): 54^{\circ} \mathrm{C}, 5.5$ and 7 min respectively; lyrebird $(7.6 \mathrm{~kb}): 52^{\circ} \mathrm{C}, 8 \mathrm{~min}$ Av10647COIIIF (23 nt): 5'-TTTGAAGCAGCAGCCTGATAYTG-3'

Av16137tProR (23 nt): 5' - ARAATRCCAGCTTTGGGAGTTGG-3'

Cytb to tRNA-Met:

Gull (6.2 kb): $53^{\circ} \mathrm{C}, 8 \mathrm{~min}$

Av15656CytbF (20 nt): 5'-AACCTGTTAGGRGAYCCAGA-3'

Av5201tMetR (20 nt): 5' - CCATCATTTTCGGGGTATGG-3'

tRNA-Leu(CUN) to $16 \mathrm{~S}$ rRNA:

Brush-turkey $(7.4 \mathrm{~kb})$ and lyrebird $(8.5 \mathrm{~kb}): 55^{\circ} \mathrm{C}$ and $54^{\circ} \mathrm{C}$ respectively, $10 \mathrm{~min}$

Both: Av13063tLeuF-LR (38 nt): 5'-TGGTCTTAGGARCCATCTATCTTGGTGCAAMTCCAAGT-3'

Brush-turkey: Av3782R16S (22 nt): 5'-CGGTCTGAACTCAGATCACGTA-3'

Lyrebird: Av3797R16S (22 nt): 5'-CGACCTGGATTTCTCCGGTCTG-3'

COIII to $16 \mathrm{~S}$ rRNA:

Turkey vulture (9.9 kb) and flycatcher (10.3 kb): Av10647COIIIF and Av3797R16S (60 $\left.{ }^{\circ} \mathrm{C}, 8 \mathrm{~min}\right)$

The reamplification products were purified by treatment with 2 units of shrimp alkaline phosphatase (SAP) and 10 units of Exonuclease I (incubated at $37^{\circ} \mathrm{C}$ for $30 \mathrm{~min}$ then $80^{\circ} \mathrm{C}$ for $15 \mathrm{~min}$ ) or were recovered from agarose gels as above. Two regions of the lyrebird mitochondrial genome were cloned: a $0.9 \mathrm{~kb}$ fragment spanning the end of tRNA-Phe plus the start of $12 \mathrm{~S}$ rRNA and a $1.4 \mathrm{~kb}$ fragment covering part of the control region, tRNA-Pro and part of NADH6. The PCR products were ligated into the pGem®-T Easy vector (Promega) and transformed into MAX Efficiency ${ }^{\circledR}$ DH5 $\alpha^{\mathrm{TM}}$ competent cells (Invitrogen) following manufacturer's instructions. Plasmid DNA was isolated from transformants containing inserts using the GenElute $^{\mathrm{TM}}$ Plasmid Miniprep Kit (Sigma). Two clones were sequenced for the first region (plus direct sequence from a PCR product generated using a specific control region primer) and three clones for the second. Sequencing reactions were run on ABI 377 or ABI 3730 sequencers and were set up according to 
manufacturer's instructions. Sequences were manually checked/corrected (including the removal of any primer sequence) and assembled using Sequencher ${ }^{\mathrm{TM}} 4.1$ (Gene Codes Corp.). Overlaps between sequences were sufficient to ensure synonymy (usually $\geq 100$ nt between individual sequences; a total of from 1 - $4 \mathrm{~kb}$ between the different long range products). Sequence identity was confirmed through Fasta searches of the EMBL database (http://www.ebi.ac.uk/fasta33/nucleotide.html).

\section{Dataset assembly}

In addition to the five new birds from this paper, 25 other complete avian mitochondrial genomes were included in the analyses (18 neognaths and seven paleognaths). The 18 neognath taxa are: chicken (Gallus gallus; GenBank accession number AP003317), Japanese quail (Coturnix japonica; AP003195), magpie goose (Anseranas semipalmata; AY309455), redhead (duck, Aythya americana; AF090337), greater whitefronted goose (Anser albifrons; AF363031), rifleman (NZ wren, Acanthisitta chloris; AY325307), grayheaded broadbill (Smithornis sharpei; AF090340), village indigobird (Vidua chalybeata; AF090341), rook (Corvus frugilegus; Y18522), peregrine falcon (Falco peregrinus; AF090338), Eurasian buzzard (Buteo buteo; AF380305), blackish oystercatcher (Haematopus ater; AY074886), ruddy turnstone (Arenaria interpres; AY074885), Oriental stork (Ciconia boyciana; AB026193), red-throated loon (Gavia stellata; AY293618), little blue penguin (Eudyptula minor; AF362763), black-browed albatross (Diomedea melanophris; AY158677) and Kerguelen petrel (Pterodroma brevirostris; AY158678). The seven paleognath taxa are: emu (Dromaius novaehollandiae; AF338711), southern cassowary (Casuarius casuarius; AF338713), great spotted kiwi (Apteryx haastii; AF338708), greater rhea (Rhea americana; Y16884), ostrich (Struthio camelus; Y12025), great tinamou (Tinamus major; AF338707) and elegant crested tinamou (Eudromia elegans; AF338710). The most up-to-date version of each GenBank file was used and the original chicken mitochondrial genome (X52392) used in previous analyses (e.g. Harrison et al. 2004, Slack et al. 2006) has been replaced by a more recent sequence.

Six reptiles were used as outgroups: American alligator (Alligator mississippiensis; Y13113), spectacled caiman (Caiman crocodylus; AJ404872), eastern painted turtle (Chrysemys picta; AF069423), green turtle (Chelonia mydas; AB012104), blue-tailed mole skink (Eumeces egregius; AB016606) and common iguana (Iguana iguana; AJ278511). This is the same set of outgroups used previously (for example, Harrison et al. 2004; Slack et al. 2006).

Two neoavian taxa (an owl and a parrot; Harrison et al. 2004) and three paleognaths (extinct NZ moas; Cooper et al. 2001, Haddrath and Baker 2001) were omitted from the full analyses until paired taxa are available. In the meantime, the owl and parrot do not affect the position of the root of the avian tree that is 
the focus of this study. We are in the process of sequencing a barn owl (Tyto), an African parrot (lovebird, Agapornis) and a forest falcon (Micrastur) (www.awcmee.massey.ac.nz/mt_genomes). Based on previous experience of improved taxon sampling (Lin et al. 2002; Delsuc et al. 2003; Harrison et al. 2004) the addition of these three mitochondrial genomes will help stabilize the position of both owls and parrots within Neoaves for future analyses. Similarly, there is some instability within paleognaths when moas are included (Slack et al. 2003), and the issue of fine-tuning paleognath interrelationships will be readdressed once additional kiwi sequences become available (G.C. Gibb, in preparation).

\section{Phylogenetic analysis}

Sequences were aligned in SeAl v1 (Rambaut 1996), at the amino acid level for protein-coding genes, and based on secondary structure for RNA genes. Each dataset has 12 protein-coding genes, two rRNAs and 21 tRNAs (lacking tRNA-Phe because it is still not available for the rifleman). Gaps, ambiguous sites adjacent to gaps, the NADH6 (light-strand encoded), and stop codons (often incomplete in the DNA sequence), were excluded from the alignment.

We made two alignments; with and without the 6 outgroup taxa. The 'birds-only' dataset was used first to study relationships within the ingroup (birds) in order to test whether there were any changes to the tree when the outgroup was added. In practice, the tree from the birds-only dataset was compared with the tree using only the birds from the full alignment (aligned including the outgroup). This allowed the separation of any effects of adding the outgroup, from any changes from the alignment. The full dataset had 11,737 unambiguously aligned nucleotide sites, the birds-only dataset has 13,440 nucleotides (15\% longer).

In previous work, RY-coding of the most variable partitions of the nucleotide data (especially the $3^{\text {rd }}$ codon position) has been shown to be advantageous (Delsuc et al. 2003; Phillips and Penny 2003; Phillips et al 2006). The recoding increases the proportion of changes on internal branches of the tree (treeness) and alleviates the differences in nucleotide composition (Relative Compositional Variability, RCV). It also increases concordance between mitochondrial and nuclear datasets. RY-coding does increase the ML scores, but because RY-coding is not strictly nested within nucleotide coding (M.A. Steel, pers. comm.) it is not valid to compare their ML scores directly. However, because of the better fit of the data to the model (higher treeness, and less variability in nucleotide composition (lower RCV), this is our preferred method of analysis of vertebrate mitochondrial data. Thus the trees reported here were inferred with the third codon positions recoded as R and Y. Analysis is by standard programs including ModelTest (Posada and Crandall 1998), PAUP* (Swofford 1998) and MrBayes (Ronquist and Huelsenbeck 2003). We ran 500 unconstrained ML bootstrap replicates with PAUP* on the Helix computing cluster 
(www.helix.massey.ac.nz), plus a partitioned Bayesian analysis using four MCMCMC chains of $10^{7}$ generations. The full data sets and command blocks for both PAUP* and MrBayes are available in nexus format at www.awcmee.massey.ac.nz/downloads. These files illustrate the procedures used both in finding optimal estimates for gamma and invariant sites for the different data partitions, as well running subsets of the data as RY coded.

\section{Results}

The five new avian complete mitochondrial genomes are deposited in GenBank under the following accession numbers: Australian brush-turkey (Alectura lathami, AY346091); superb lyrebird (Menura novaehollandiae, AY542313); fuscous flycatcher (Cnemotriccus fuscatus, AY596278); southern blackbacked gull (Larus dominicanus, AY293619); and turkey vulture (Cathartes aura, AY463690). The gull, brush-turkey and turkey vulture have the standard avian gene order first identified in the chicken (Desjardins and Morais 1990). This is expected in that the other galliforms in the dataset (chicken and quail) and the two other shorebirds (oystercatcher and turnstone) are already known to have the standard gene order. However, the turkey vulture also appears to have the standard avian gene order, and thereby differs from the two falconiform mitochondrial genomes (falcon and buzzard) already available. By itself, this is certainly of interest, but not sufficient yet to exclude it from the falconiforms, even though for characters with an extremely high number of states parsimony is an ML estimator (Steel and Penny 2004).

The lyrebird and flycatcher genomes have the alternative avian gene order first recognized by Mindell et al. (1998). The flycatcher result is as expected; Mindell et al. (1998) examined nine major suboscine lineages (including a tyrannid flycatcher) and found that all had the alternative gene order. On the other hand, Mindell et al. (1998) found that a set of 106 oscine species had the standard avian gene order. However, that set was mostly Passeridae (90 taxa) and Fringillidae (11 taxa) and the remaining five families (Corvidae, Sturnidae, Cisticolidae, Certhiidae, Estrildidae) were each represented by a single taxon. Although Mindell et al. (1998) concluded "that the alternative mitochondrial gene orders distinguish the two primary groups of songbirds (order Passeriformes), oscines and suboscines” Bensch and Härlid (2000) subsequently identified the alternative gene order in an oscine group (Phylloscopus warblers, Sylviidae). The lyrebird (Menuridae) now provides the second example of the alternative gene order in oscine passerines. The duplicated control region associated with the alternative gene order has been reduced to a short non-coding region in both the lyrebird and the Phylloscopus warblers. However, if this non-coding region is still similar to the control region then the sequence fragment (most of NADH6, tRNA-Glu, part of the control region) that Mindell et al. (1998) used to examine most of their oscine species (102 of 106) might not detect the alternative gene order when it is present. This could happen, for example, where the 
rearrangement is recent, or if a form of concerted gene evolution homogenized the duplicates (G.C. Gibb et al. in prep.).

\section{Unrooted tree}

We know from both simulations (Holland et al. 2003) and empirical studies on mammals (Lin et al. 2002) and birds (Slack et al. 2003) that an outgroup can disrupt a previously established ingroup tree (see Introduction). Therefore we first report an unrooted ML tree for the 30 bird sequences, together with the results of 500 full ML bootstrap runs. The data is the combined protein coding and RNA genes for both datasets - aligned with or without the 6 outgroup taxa, giving a combined length of 11,737 and 13,440 nucleotides respectively. Again, we find that re-coding the $3^{\text {rd }}$ position (cdp) as R/Y characters markedly reduces the relative compositional variability ( $\mathrm{RCV})$, and increases the signal on the internal branches compared with the external branches (the treeness value is increased). As before, the models were optimized separately for each of the four data partitions using ModelTest (Posada and Crandall 1998). The four partitions were: codons 1\&2; codon 3; RNA stems; and RNA loops. The unrooted maximum likelihood tree for the birds-only dataset is shown in Figure 1.

The position of the megapode (brush turkey) is, as expected, a deep lineage in Galliformes, and the three galliforms (chicken, quail and brush-turkey) then group with the three anseriforms (duck, goose, and magpie goose [Anseranas]), forming Galloanserae. The tyrant flycatcher, as expected, joins with the other suboscine (broadbill) and their union is quite deep. Similarly, the lyrebird is deeper in the oscines than the indigo bird/rook divergence. It is worth noting that with the new oscine and suboscine included that the NZ wren (rifleman) now comes basal to the passerines. Overall, the results strongly support the revised classification of oscines (Ericson et al. 2002b) into Menurae (which includes lyrebird) and Euoscines (which includes rook and indigo bird). These three new taxa (brush turkey, tyrant flycatcher and lyrebird) were selected from prior knowledge in anticipation that they would break up long branches that could be attracting the outgroup (a long-branch attraction problem, Hendy and Penny 1989) when rooting the avian tree. This will be re-examined later when examining the position of the root.

The gull joins strongly with the turnstone, rather than with the other shorebird (oyster catcher). More formally, the Lari (gulls) are closer to the Scolopaci (turnstones) than to the Charadrii (oyster catcher). The result is in agreement with the results from nuclear data of Ericson et al. (2003) and Paton et al. (2003). It is not in agreement with Sibley and Ahlquist (1990), but it is only a single interchange (nearest neighbor interchange) on the tree. We say that a tree is 'locally stable' if taxa shift no more than one branch on the 
tree (Cooper and Penny, 1997). Again, the agreement of nuclear and mitochondrial data is pleasing, but expected.

The fifth new species is the turkey vulture - a New World vulture. The two issues here are whether the falconiforms (in a broad sense) are monophyletic, and if not, whether the turkey vulture goes with the stork. On the present dataset, turkey vulture does not come with the two other falconiforms, but nor is it particularly close to the stork. Thus there is some support for the independent origin of core falconiforms and New World vultures, but we need a wider selection of taxa, such as flamingos, grebes, herons, rails, etc. before firmly identifying the closest relative of New World vultures. However, it is also desirable to have additional core falconiforms, because the falcon still has a tendency to go deeper in the tree, disrupting the falcon/buzzard group. Similarly, Fain and Houde (2004) do not recover the falcon/buzzard clade, emphasizing the need for additional taxa. On the present data set the falconiforms (in the broad sense, including New World vultures) are not monophyletic, but we cannot distinguish yet between their being polyphyletic (diphyletic in this case) or paraphyletic. This latter case is potentially interesting from the ecological/life histories viewpoint in that many of the sea- and shorebirds may have diversified from carnivores (raptors) into a related niche in a marine environment. At present, the position of both the peregrine falcon and the stork are still locally unstable and improved taxon sampling is thus required.

\section{Rooted tree}

The incorporation of the brush turkey, the tyrant flycatcher and the lyrebird breaks up long unbranched edges situated deep in the avian tree. Therefore the next step is to identify what (if any) effect this has for locating the position of the root in the avian tree. Figure 2 is the rooted tree, using the six taxa outgroup constituted of two crocodilians, two turtles and two lizards. This reptilian outgroup joins with strong support between paleognaths and neognaths (Figure 2), and is thus consistent with most recent work on both nuclear genes (van Tuinen et al. 2000; Chubb 2004; Garcia-Moreno et al. 2003) and mitochondrial genomes (Paton et al. 2002; Harrison et al. 2004). Of the outgroup taxa, crocodilians are again closest to birds.

An important observation is that the addition of the outgroup does not make major disruptions to the ingroup tree as was previously the case with fewer taxa (Slack et al. 2003). As mentioned earlier, simulations (Holland et al. 2003) have shown that the addition of the outgroup can lead to disruption of the previously established relationships within the ingroup as it has been observed in placental mammal mitogenomic trees for example (Lin et al. 2002). It is therefore pleasing that the basic ingroup tree (from the unrooted tree of Figure 1) is not altered when the outgroup is included, giving us more confidence in 
the present rooting. As a result, the paleognath/neognath division is supported as well as the strict monophyly of passerines. As before, there is increased support for the chicken/duck grouping (Galloanseriforms), again in agreement with morphological (Cracraft 2001), nuclear (van Tuinen et al. 2000; Chubb 2004; Garcia-Moreno et al. 2003) and mitochondrial (Paton et al. 2002; Harrison et al. 2004) datasets. Within the Neoaves, there is strong support for Passeriformes occupying a basal position, and the Conglomerati (Cracrafti) still holds up, though significant groups such as rails and pigeons are not yet represented and there are differences in predictions between Cracraft (2001) and Cracraft et al. (2004). There is one interesting difference between the birds-only results (Figure 2), and the tree derived from the alignment that includes the 6 outgroup taxa (Figure 1). This is that the first divergence is between shorebirds and raptors/ seabirds on the birds-only dataset, but the falconiforms (buzzard and falcon in particular) are deeper on the tree from the alignment containing the 6-taxon outgroup. This difference, especially the deeper divergence of shorebirds is worth following up, given the comment of Feduccia (2006) that shorebirds appear to be a late Cretaceous lineage, and from the results of Slack et al (2006) that discusses the agreement here between estimates of times of divergence from molecular data and existence of early fossil footprints of birds. However, some relationships within the Conglomerati remain unresolved with low bootstrap values except for the monophyly of Charadriiformes and for the putative grouping of Procellariformes with Gaviiformes, Sphenisciformes and Ciconiiformes (Figures 2 and 3). The lack of conflicting signal for this latter grouping (see Figure 3) is interesting in that it argues against there being any strong systematic bias (Phillips et al. 2004).

\section{Increased stability from breaking up long branches (edges).}

A primary interest here is testing whether the improved taxon sampling (breaking up long branches within the avian tree) leads to improved stability. Qualitatively it appears so since the addition of brush turkey, tyrant flycatcher and lyrebird, has significantly increased the strength of the rooting between paleognaths and neognaths. To make this conclusion quantitative we deleted each of the 30 avian taxa in turn, and ran 100 bootstrap samples on each of the reduced 30 data sets, using ML. Thus each dataset had 29 birds and the 6 outgroup taxa. This is essentially a jackknife approach (Lanyon 1985), deleting taxa sequentially and measuring the effect (Penny and Hendy 1985). For the 30 bootstrap runs, the outgroup was constrained so that the six outgroup taxa always grouped together, as well as the two turtles, the two crocodilians and the two lizards. There were no constraints on the avian tree. For each of the 30 datasets the percentage of trees from the 100 bootstrap samples were summed under the categories of paleo/neognath rooting, rooting within paleognaths, galloanseriform rooting, passerine rooting, or within the Neoaves generally (Table 1). For both the galloanseriform and passerine cases the results include the rooting within, for example, galloanseriforms or between galloanseriforms and all other birds. 
With phylogenetic trees (including our own studies) it is traditional to give the results first and subsequently think up explanations! Here we reverse the process and give our predictions first (Table 1, column 2) and then ran the bootstrap samples before comparing the predictions and the results. Because our theme has been that breaking up long branches improves the stability of the tree, we focus our predictions on how we expect the additional sequences to affect the bootstrap values for different rootings. The second column in Table 1 indicates our predictions on how the bootstrap values might change for each jackknife sample, relative to the bootstrap values with the full 30 bird dataset. The predictions are whether we expect that removal of a particular avian taxon will increase the bootstrap values for a competing placement of the root. For example, we expect that removing the brush turkey will lead to the root appearing significantly more often on the galloanseriform lineage, indicated as ' $>\mathrm{Ga}$ '. Concomitably, there would have to be a reduction in cases where the root comes into the expected position between paleognaths and neognaths. The magnitude of the effect is hard to predict, but based on prior experience (for example, Slack et al. 2003 ) we would expect bootstrap values to decrease by 10-30\%, and possibly even more. Where it seemed that a smaller effect could occur we have added question marks, for example '>Ga??'. Note that to estimate the increased stability from adding a taxon, we are measuring the decrease in bootstrap values from removing the taxon. More than one value can occur in a column, for example in column 3, the first value is the paleognaths occurring as a monophyletic group; the second for the neognaths. Similarly, within the galloanseriforms, the root could come on the branch basal to the group, or within the chicken or the duck group. One aberrant bootstrap sample could affect several internal branches on the tree. For example, a ' $1+1+1$ ' will usually be one bootstrap sample that changes three internal branches. Having given our predictions, the actual results are given in Table 1.

Surprisingly, our predictions were relatively poor; there was virtually no loss of stability by removing any individual taxon. That is, the results were considerably more robust than we expected. Overall this implies that there is some cumulative effect from adding a range of taxa that break up long branches, leading to the increased stability. Nevertheless there are interesting individual effects especially evidenced when the rows are summed to give the 'total' effect of removing each taxon. Removal of the New Zealand wren (rifleman) had the largest effect, by which we infer that adding this taxon into the dataset contributed the most to stabilizing the tree. As expected, removing a deeper branching taxon had more effect than removing one member of a pair of more closely related taxa. For example, removing the brush turkey had more effect than deleting either the chicken or quail. Overall, removal of a passerine had a relatively large effect, a result that might be explained by their higher evolutionary rates. However, we were surprised that the removal of the penguin has also a significant effect, despite its relatively short branch and its internal 
position in the avian tree. We had assumed that the problem was that the stork was relatively unstable on the tree, but we must consider penguin as a potentially difficult taxon.

In an additional analysis, we computed a Z-closure super-network (Huson et al. 2004), using SplitsTree 4.1 (Huson and Bryant 2006), from the 30 jackknife ML trees where each avian species was omitted in turn from the rooted tree (Figure 3). This method offers a graphical summary of the topologies of the 30 jackknifed trees and allows identification of the parts of the phylogeny which were in agreement for all the 30 trees with 29 avian taxa. Areas of conflict are represented as rectangles in the super-network, reflecting any instability caused by removing individual taxa. The results in Figure 3 are striking in that there is no incongruence detected with respect to the position of the root of the avian part of the tree. This again shows the lack of effect of removing any single taxon and illustrates that a robust rooting of the avian tree can be obtained by using an expanded number of mitochondrial genomes. In contrast, uncertainty is detected in three places within the avian tree corresponding to areas of instability identified previously (see Figure 2). The first is between the tinamou and the deeper ratites (ostrich and rhea) where the tree is not completely stable and therefore removing taxa has an effect. Similarly, there is a tendency for the rifleman to come onto the basal suboscine branch as represented by a cycle in Figure 3. However, the major effect was inside the Conglomerati (Cracrafti) with a series of boxes involving the raptors (including turkey vulture) and the sea birds and shore birds. Either the core raptors (falcon and buzzard) or the shore birds can come out basal in this Conglomerati group. This relatively unstable part of the avian tree is being studied further by sequencing additional mitochondrial genomes, including osprey, a forest falcon, and additional potential relatives of stork and penguin. However, although the variability within the Conglomerati (Cracrafti) is very interesting, it is not relevant to the question at hand of demonstrating the stability of the root of the avian tree.

\section{Discussion}

The long-term goal of this project is to use nuclear and mitochondrial sequences, together with fossil data, to test modes of macroevolution in the Late Cretaceous (Penny and Phillips 2004). The primary aim of this study is to determine whether the breaking up of some long branches on the avian tree leads to agreement between nuclear and mitochondrial data on the position of the root. In this respect, the position of the root now appears in agreement between morphological, nuclear and mitochondrial data, and thus it is time to move on to other questions. Data from complete mitochondrial genomes obviously takes longer to obtain for each taxon than sequences from a single nuclear gene. This has lead to some false starts concerning the position of the avian root due in part to incomplete taxon sampling (Härlid and Arnason 1999; Mindell et al. 1999), though earlier analyses by Braun and Kimball (2002) and Slack et al. (2003) indicated that the 
paleognath/neognath division could not be rejected. There appears to have been an 'urban myth' that mitochondrial genomes could not recover the same avian root as morphological and nuclear data. In fact, the main problem was incomplete taxon sampling, a problem that is better solved by more data collection than by polemics. Indeed, it is hard to imagine theoretical reasons that would lead to different roots from different datasets. It is unlikely that the trees would be fundamentally different and therefore additional sequences and better methods of analysis are a more likely solution to the problem. We should all aim at improving data sets to test whether they lead to consensus.

With the major early divisions having been resolved, perhaps the next step on the avian tree is to provide resolution within Neoaves and especially within the Conglomerati (Cracrafti). For this specific question, breaking up long branches does appear to be an effective strategy, but obviously depends on appropriate taxa being available. When there was only a single suboscine (broadbill) and a single Falconiform (falcon) in the dataset, there was a strong tendency for them to come together (see discussion in Slack et al. 2003). However, with additional passerine being incorporated, we now have strong support for the respective monophyly of both Passeriformes and Conglomerati. The falcon and the stork have both been difficult to place in the mitogenomic tree in that both still appear to be uncertain about their final position. In early datasets, the falcon could even occur at the base of the passerines (Slack et al. 2003). The addition of the buzzard has reduced the wandering of the falcon, but the falcon/buzzard grouping is relatively weak, and the falcon can go deeper within the neoaves. We are currently sequencing a forest falcon (Micrastur) to determine its effect because in Sibley and Ahlquist (1990) this was the deepest divergence among relatives of the falcon. The present data set has no species from within the proposed Metaves group of Fain and Houde (2004), and thus we cannot yet comment on that hypothesis. Mitochondrial genomes from members of that group are being completed and then we will be able to test the predicted distinction of Neoaves into Metaves and Coronaves.

The problem of the early divisions of Neoaves is going to be difficult. Cracraft (2001) proposed six unresolved groups, and this increased to nine in Cracraft et al. (2004). It has been suggested that the early divergence of neoavian birds was an 'explosive radiation' (as just one example, see Poe and Chubb 2004). However, for a radiation to be 'explosive' it requires both a rapid series of lineage divergences, combined with simultaneous morphological and/or ecological adaptations. It would not be sufficient just for divergences of lineages to be close together - that could occur very easily by a rapidly dispersing taxon even though the subsequent ecological and morphological divergences occurred many millions of years later. Such a delayed adaptation would scarcely be an 'explosive' radiation. The first aspect (rate of diversification of lineages) is best studied from molecular data (as is done here). The second, the timing of 
adaptations to new environments/niches is perhaps better studied from the fossil record. As yet, in the present data we do not see any evidence whatsoever for an 'explosive radiation' of neoavian birds, even though the early divergences may have been relatively rapid in Neoaves. We are more cautious, and want to see data on the speed of morphological and ecological changes before coming to such dramatic conclusion about an explosive radiation. We prefer at present to consider the early diversification of Neoaves as an adaptive radiation, indicating that it is a relatively fast radiation but strictly according to known microevolutionary principles.

Indeed, as mentioned earlier, one of our main goals is to determine the extent to which the processes of microevolution are sufficient to explain macroevolution. This is the theme behind our models of avian and mammal diversification in the Late Cretaceous (Penny and Phillips 2004), and whether (by comparing the human and chimpanzee genomes) there is any aspect of the human genome that is not a normal microevolutionary (genetic) process (Penny 2004). We think that the 'explosive radiation' should be to restrict to possible cases where normal microevolutionary process are clearly insufficient to account for macroevolution. To test such hypotheses we need a tree sufficiently stable in order that good timing estimates are possible, preferably on combined nuclear and mitochondrial data. All progress in this direction is welcomed.

\section{Acknowledgements}

We especially thank Dick Gill, Darryl Jones, Ian Owens, Cathy Nock, Fred Sheldon and Donna Dittman for the samples. Tim White and Warwick Allen assisted with computer programs for running analyses on the Helix supercomputer and Gillian Gibb for considerable assistance in manuscript preparation. F.D. acknowledges the support of a Lavoisier Postdoctoral Grant from the French Ministry of Foreign Affairs. Both the New Zealand Marsden Fund and the Centre for Research Excellence funds supported the work. 


\section{References}

Barker, F.K., Barrowclough, G.F., Groth, J.G., 2002. A phylogenetic hypothesis for passerine birds: taxonomic and biogeographic implications of an analysis of nuclear DNA sequence data. Proc. R. Soc. Lond. B 269, 295-308.

Barker, F.K., Cibois, A., Schikler, P., Feinstein, J., Cracraft, J., 2004. Phylogeny and diversification of the largest avian radiation. Proc. Natl. Acad. Sci. U.S.A. 101, 11040-11045.

Bensch, S., Härlid, A., 2000. Mitochondrial genomic rearrangements in songbirds. Mol. Biol. Evol. 17, 107-113.

Braun, E.L, Kimball, R.T., 2002. Examining basal avian divergences with mitochondrial sequences: Model complexity, taxon sampling, and sequence length. Syst. Biol. 51, 614-625.

Buckley, T.R., 2002. Model misspecification and probabilistic tests of topology: Evidence from empirical data sets. Syst. Biol. 51, 509-523.

Chang, J.T., 1996. Inconsistency of evolutionary tree topology reconstruction methods when substitution rates vary across characters. Math. Biosc. 134, 189-215.

Chubb, A.L., 2004. New nuclear evidence for the oldest divergence among neognath birds: the phylogenetic utility of ZENK (i). Mol. Phylogen. Evol. 30, 140-151.

Cooper, A., Lalueza-Fox, C., Anderson, S., Rambaut, A., Austin, J., Ward, R., 2001. Complete mitochondrial genome sequences of two extinct moas clarify ratite evolution. Nature 409, 704-707.

Cooper, A., Penny, D., 1997. Mass survival of birds across the Cretaceous-Tertiary boundary: Molecular evidence. Science 275, 1109-1113.

Cracraft, J., 2001. Avian evolution, Gondwana biogeography and the Cretaceous-Tertiary mass extinction event. Proc. Roy. Soc. Lond. B 268, 459-469.

Cracraft, J., Barker, F.K., Braun, M. and 11 others, 2004. Phylogenetic relationships among modern birds (Neornithes). In: J. Cracraft and M.J. Donoghue (Eds), Assembling the Tree of Life. Oxford Univ. Press, pp 468-489.

Delsuc, F., Phillips, M.J., Penny, D., 2003. Comment on "Hexapod origins: monophyletic or paraphyletic?" Science 301, 1482d.

Desjardins, P., Morais, R., 1990. Sequence and gene organization of the chicken mitochondrial genome - a novel gene order in higher vertebrates. J. Mol. Biol. 212, 599-634.

Edwards, S.V., Boles, W. E., 2002. Out of Gondwana: the origin of passerine birds. Trends Ecol. Evol. 17, 347-340.

Edwards, S.V., Fertil, B., Giron, A., Deschavanne, P.J., 2002. A genomic schism in birds revealed by phylogenetic analysis of DNA strings. Syst. Biol. 51, 599-613. 
Ericson, P.G.P., Christidis, P.L., Cooper, A., Irestedt, M., Jackson, J., Johansson, U.S., Norman, J.A., 2002a. A Gondwanan origin of passerine birds supported by DNA sequences of the endemic New Zealand wrens. Proc. Roy. Soc. Lond. B 269, 235-241.

Ericson, P.G.P., Christidis, P.L., Irestedt, M., Norman, J.A., 2002b. Systematic affinities of the lyrebirds (Passeriformes: Menura), with a novel classification of the major groups of passerine birds. Mol. Phylogenet. Evol. 25, 53-62.

Ericson, P.G.P., Johansson, U.S., 2003. Phylogeny of Passerida (Aves: Passeriformes) based on nuclear and mitochondrial sequence data. Mol. Phylogenet. Evol. 29, 126-138.

Ericson, P.G.P., Envall, I., Irestedt, M., Norman, J.A., 2003. Inter-familial relationships of the shorebirds (Aves: Charadriiformes) based on nuclear DNA sequence data. BMC Evol. Biol. 3, 16.

Fain M.G., Houde, P., 2004. Parallel radiations in the primary clades of birds. Evolution 58, 2558-2573.

Feduccia, A., 2006. Mesozoic aviary takes form. Proc. Natl Acad. Sci. USA 103, 5-6.

Garcia-Moreno, J., Mindell, D.P., 2000. Rooting a phylogeny with homologous genes on opposite sex chromosomes (gametologs): a case study using avian CHD. Mol. Biol. Evol. 17, 1826-1832.

Garcia-Moreno, J., Sorenson, M.D., Mindell, D.P., 2003. Congruent avian phylogenies inferred from mitochondrial and nuclear DNA sequences. J. Mol. Evol. 56, 1-11.

Haddrath, O., Baker, A.J., 2001. Complete mitochondrial DNA genome sequences of extinct birds: ratite phylogenetics and the vicariance biogeography hypothesis. Proc. R. Soc. Lond. B 268, 939-945.

Haring, E., Kruckenhauser, L., Gamauf, A., Riesing, M.J., Pinsker, W., 2001. The complete sequence of the mitochondrial genome of Buteo buteo (Aves, Accipitridae) indicates an early split in the phylogeny of raptors. Mol. Biol. Evol. 18, 1892-1904.

Härlid, A., Arnason, U., 1999. Analyses of mitochondrial DNA nest ratite birds within the "Neognathae" supporting a neotenous origin of ratite morphological characters. Mol. Phylogenet. Evol. 12, 115-123.

Härlid, A., Janke, A., Arnason, U., 1999. The complete mitochondrial sequence of Rhea americana and early avian divergences. J. Mol. Evol. 46, 669-679.

Harrison, G.L., McLenachan, P.A., Phillips, M.J., Slack, K.E., Cooper, A., Penny, D., 2004. Four new avian mitochondrial genomes help get to basic evolutionary questions in the Late Cretaceous. Mol. Biol. Evol. 21, 974-983

Helbig, A.J., Seibold, I. 1996. Are storks and New World vultures paraphyletic? Mol. Phylog. Evol. 6, 315-319.

Hendy, M.D., Penny, D., 1989. A framework for the quantitative study of evolutionary trees. Syst. Zool. 38, 297-309.

Holland, B.R., Penny, D., Hendy, M.D., 2003. Outgroup misplacement and phylogenetic inaccuracy under a molecular clock: A simulation study. Syst. Biol. 52, 229-238. 
Huson, D.H., Dezulian, T., Kloepper, T., Steel, M.A., 2004. Phylogenetic super-networks from partial trees. IEEE/ACM Trans. Comput. Biol. Bioinform. 1, 151-158.

Huson D.H., Bryant D., 2006. Application of phylogenetic networks in evolutionary studies. Mol. Biol. Evol. 23, 254 - 267.

Irestedt, M., Johansson, U.S., Parsons, T.J., Ericson, P.G.P., 2001. Phylogeny of major lineages of suboscines (Passeriformes) analysed by nuclear DNA sequence data. J. Avian Biol. 32, 15-25.

Kocher, T.D., Thomas, W.K., Meyer, A., Edwards, S.V., Pääbo, S., Villablanca, F.X. and Wilson, A.C., 1989. Dynamics of mitochondrial DNA evolution in animals: amplification and sequencing with conserved primers. Proc. Natl Acad. Sci. USA 86, 6196-6200.

Lanyon, S.C., 1985. Detecting internal inconsistencies in distance data. Syst. Zool. 34, 397-403.

Lin, Y.-L., McLenachan, P.A., Gore, A.R., Phillips, M.J., Ota, R., Hendy, M.D., Penny, D., 2002. Four new mitochondrial genomes, and the stability of evolutionary trees of mammals. Mol. Biol. Evol. 19, 2060-2070.

Livezey, B.C., Zusi, R.L., 2001. Higher-order phylogenetics of modern Aves based on comparative anatomy. Netherlands J. Zool. 51, 179-205.

Mayr, G., Clarke, J., 2003. The deep divergences of neornithine birds: a phylogenetic analysis of morphological characters. Cladistics 19, 527-553.

Mindell D.P., Sorenson, M.D., Dimcheff, D.E., 1998. Multiple independent origins of mitochondrial gene order in birds. Proc. Natl Acad. Sci. USA 95, 10693-10697.

Mindell D.P., Sorenson, M.D., Dimcheff, D.E., Hasegawa, M., Ast, J.C., Yuri, T., 1999. Interordinal relationships of birds and other reptiles based on whole mitochondrial genomes. Syst. Biol. 48, 138152.

Paton, T., Haddrath, O., Baker, A.J., 2002. Complete mitochondrial DNA genome sequences show that modern birds are not descended from transitional shorebirds. Proc. Roy. Soc. Lond. B 269, 839-846.

Paton, T., Baker, A.J., Groth, J.G., Barrowclough, G.F., 2003. RAG-1 sequences resolve phylogenetic relationships within Charadriiform birds. Mol. Phylogenet. Evol. 29, 268-278.

Penny, D., 2004. Our relative genetics. Nature 427, 208-209.

Penny, D., Foulds, L.R., Hendy, M.D., 1982. Testing the theory of evolution by comparing phylogenetic trees constructed from five different protein sequences. Nature 297, 197-200.

Penny, D., Hendy, M.D., 1985. The use of tree comparison metrics. Syst. Zool. 34, 75-82.

Penny, D. Phillips, M.J., 2004. The rise of birds and mammals: Are microevolutionary processes sufficient for macroevolution. Trends Ecol. Evol. 19, 516-522.

Phillips, M. J., Delsuc, F., D. Penny, D., 2004. Genome-scale phylogeny: sampling and systematic errors are both important. Mol. Biol. Evol. 21, 1455-1458. 
Phillips, M.J., Penny, D., 2003. The root of the mammalian tree inferred from whole mitochondrial genomes. Mol. Phylogenet. Evol. 28, 171-185.

Phillips, M.J., McLenachan, P.A., Gibb, G.C., Penny, D., 2006. Combined nuclear and mitochondrial protein-coding DNA sequences resolve the interrelations of the major Australasian marsupial radiations. Syst. Biol. 55, 122-137.

Poe, S., Chubb, A.L., 2004. Birds in a bush: Five genes indicate explosive evolution of avian orders. Evolution 58, 404-415.

Posada, D., Crandall, K.A., 1998. Modeltest: testing the model of DNA substitution. Bioinformatics 14, 817-818.

Rambaut, A., 1996. Se-Al: Sequence Alignment Editor. Available at http://evolve.zoo.ox.ac.uk/.

Rea, A.M., 1983. Cathartid affinities: a brief overview. In: Wilbur, S.R., Jackson, J.A. (Eds), Vulture biology and management. University of California Press, Berkeley, California, pp 26-54.

Reyes, A., Gissi, C., Catzeflis, F., Nevo, E., Pesole, G., Saccone, C., 2004. Congruent mammalian trees from mitochondrial and nuclear genes using Bayesian methods. Mol. Biol. Evol. 21, 397-403.

Ronquist, F., Huelsenbeck, J. P., 2003. MrBayes3: Bayesian phylogenetic inference under mixed models. Bioinformatics 19, 1572-1574.

Seibold, I., Helbig, A. J., 1995. Evolutionary history of New and Old World vultures inferred from nucleotide sequences of the mitochondrial cytochrome $b$ gene. Phil. Trans. Roy. Soc. Lond. B 350, 163-178.

Sibley, C.G., Ahlquist, J. E., 1990. Phylogeny and classification of birds. Yale University Press, New Haven, CT.

Slack, K.E., Janke, A., Penny, D., Arnason, U., 2003. Two new avian mitochondrial genomes (penguin and goose) and a summary of bird and reptile mitogenomic features. Gene. 302, 43-52.

Slack, K.E., Jones, C.M., Tatsuro, A., Harrison, G.L., Fordyce, R.E., Arnason, U., Penny, D., 2006. Early penguin fossils, plus mitochondrial genomes, calibrate avian evolution. Mol. Biol. Evol. 23, (in press, available on line)

Sorenson M.D., Ast, J.C., Dimcheff, D.E., Yuri, T., Mindell, D.P., 1999. Primers for a PCR-based approach to mitochondrial genome sequencing in birds and other vertebrates. Mol. Phylogenet. Evol. $12,105-114$.

Steel, M.A., Penny, D., 2004. Two further links between MP and ML under the Poisson model. Appl. Math Lett. 17, 785-790

Swofford, D.L., 1998. PAUP*: Phylogenetic Analysis Using Parsimony (*and other methods). Sinauer Associates Sunderland, Massachusetts. 
Thomas, G.H., Wills, M.A. Székely, T., 2004. Phylogeny of shorebirds, gulls, and alcids (Aves: Charadri) from the cytochrome c gene: parsimony, Bayesian inference, minimum evolution and quartet puzzling. Mol. Phyl. Evol. 30, 516-526

Van Tuinen, M., Sibley, C. Hedges, S. B., 2000. The early history of modern birds inferred from DNA sequences of nuclear and mitochondrial ribosomal genes. Mol. Biol. Evol. 17, 451-457.

Van Tuinen, M., Waterhouse, D., Dyke, G.J., 2004. Avian molecular systematics on the rebound: a fresh look at modern shorebird phylogenetic relationships. J. Avian Biol. 35, 191-194. 
Table 1. Predicted and actual effects on the position of the root from taxon removal

\begin{tabular}{|c|c|c|c|c|c|c|c|}
\hline species omitted & predicted & $\begin{array}{c}\text { paleo/ } \\
\text { neognaths } \ddagger\end{array}$ & $\begin{array}{c}\text { Paleognaths } \\
\mathrm{Pg} \\
\end{array}$ & $\begin{array}{c}\text { Galloanseres } \\
\mathrm{Ga} \\
\end{array}$ & $\begin{array}{c}\text { Passerines } \\
\mathrm{Pa} \\
\end{array}$ & Neoaves & Total \\
\hline None, all 30 birds & n.a. & & & & & & \\
\hline emu & - & $100 / 99$ & - & 1 & - & - & 1 \\
\hline cassowary & - & $99 / 97$ & - & $2+1$ & $1+1+1$ & - & 6 \\
\hline kiwi & - & $100 / 97$ & - & $1+1$ & 1 & 1 & 4 \\
\hline rhea & $>\operatorname{Pg} ? ?$ & $100 / 95$ & - & $1+1$ & $3+4 \times 1$ & 4 & 13 \\
\hline ostrich & $>\operatorname{Pg} ? ?$ & $100 / 93$ & - & $3+1$ & $3+1$ & 4 & 12 \\
\hline tinamou & $>\mathrm{Pg}$ & $100 / 91$ & - & 1 & $2+2+2$ & 8 & 15 \\
\hline gs_tinamou & $>\mathrm{Pg}$ & $100 / 95$ & - & $3+1$ & $1+1+1$ & 2 & 9 \\
\hline magpie goose & $>\mathrm{Ga}$ & $100 / 94$ & - & 3 & $1+1$ & 3 & 8 \\
\hline goose & - & $100 / 98$ & - & $1+1$ & $1+1+1$ & 1 & 6 \\
\hline duck & - & $99 / 96$ & 1 & 2 & - & 2 & 5 \\
\hline brush turkey & $>\mathrm{Ga}$ & $100 / 92$ & - & $7+3$ & - & 1 & 11 \\
\hline chicken & - & $100 / 99$ & - & $1+1$ & - & - & 2 \\
\hline quail & - & $100 / 97$ & - & 2 & $1+1$ & 1 & 5 \\
\hline rifleman & $>\mathrm{Pa} ?$ & $100 / 91$ & - & 2 & $5+5+3$ & 7 & 22 \\
\hline broadbill & $>\mathrm{Pa}$ & $100 / 94$ & - & $3+1$ & 2 & 3 & 9 \\
\hline flycatcher & $>\mathrm{Pa}$ & 98/96 & 2 & - & $2+1+1$ & 4 & 10 \\
\hline lyrebird & $>\mathrm{Pa}$ & 99/93 & 1 & $3+1$ & $3+2+2$ & 4 & 16 \\
\hline rook & $>\mathrm{Pa} ? ?$ & $100 / 94$ & - & $4+3 \times 1$ & $1+1$ & 2 & 11 \\
\hline indigobird & $>\mathrm{Pa} ? ?$ & $100 / 93$ & - & $3+1$ & $1+2 \times 1 *$ & 4 & 11 \\
\hline falcon & - & 99/97 & 1 & $3+2$ & - & - & 6 \\
\hline buzzard & >Oth?? & $100 / 96$ & - & - & $2+1+1$ & 4 & 8 \\
\hline turkey vulture & - & 99/94 & - & $2+1$ & 1 & 4 & 8 \\
\hline oystercatcher & - & $100 / 95$ & - & $2+1$ & 1 & 3 & 7 \\
\hline turnstone & - & $100 / 97$ & - & $2+3 \times 1$ & 1 & 1 & 7 \\
\hline gull & - & $99 / 98$ & 1 & $1+1+1$ & - & - & 4 \\
\hline stork & - & $100 / 98$ & - & 1 & 1 & 1 & 3 \\
\hline penguin & - & $100 / 91$ & - & $4+1$ & $3+1+1$ & 5 & 15 \\
\hline albatross & - & $100 / 98$ & - & 1 & - & 1 & 2 \\
\hline petrel & - & $100 / 95$ & - & 2 & - & 3 & 5 \\
\hline loon & - & $100 / 95$ & - & 3 & $1+1$ & 2 & 7 \\
\hline
\end{tabular}

\$Expected position of the root from nuclear and morphological data.

**crested tinamou joins rhea, inside ratites 
Figure Captions

Figure 1. Unrooted tree on complete mitochondrial genomes from 30 birds. The $3^{\text {rd }}$ codon position was RY coded with other sites $\left(1^{\text {st }}\right.$ and $2^{\text {nd }}$ positions for protein genes, stems and loops for RNA genes) remained as nucleotides. Bootstrap values are from 500 runs.

Figure 2. Rooted tree using six outgroup taxa. The same coding as for Figure 1. Bootstrap values are shown; * is $100 \%$.

Figure 3. Phylogenetic super-network obtained by applying the Z-closure method to the 30 jackknife ML trees where each individual bird taxon has been removed in turn from the complete dataset. This figure represents a graphical summary of multiple phylogenies reconstructed from the different sets of taxa. It shows on which parts of the phylogeny all partial trees are in agreement (dichotomous parts) and where there exist contradicting signals (networking parts). 


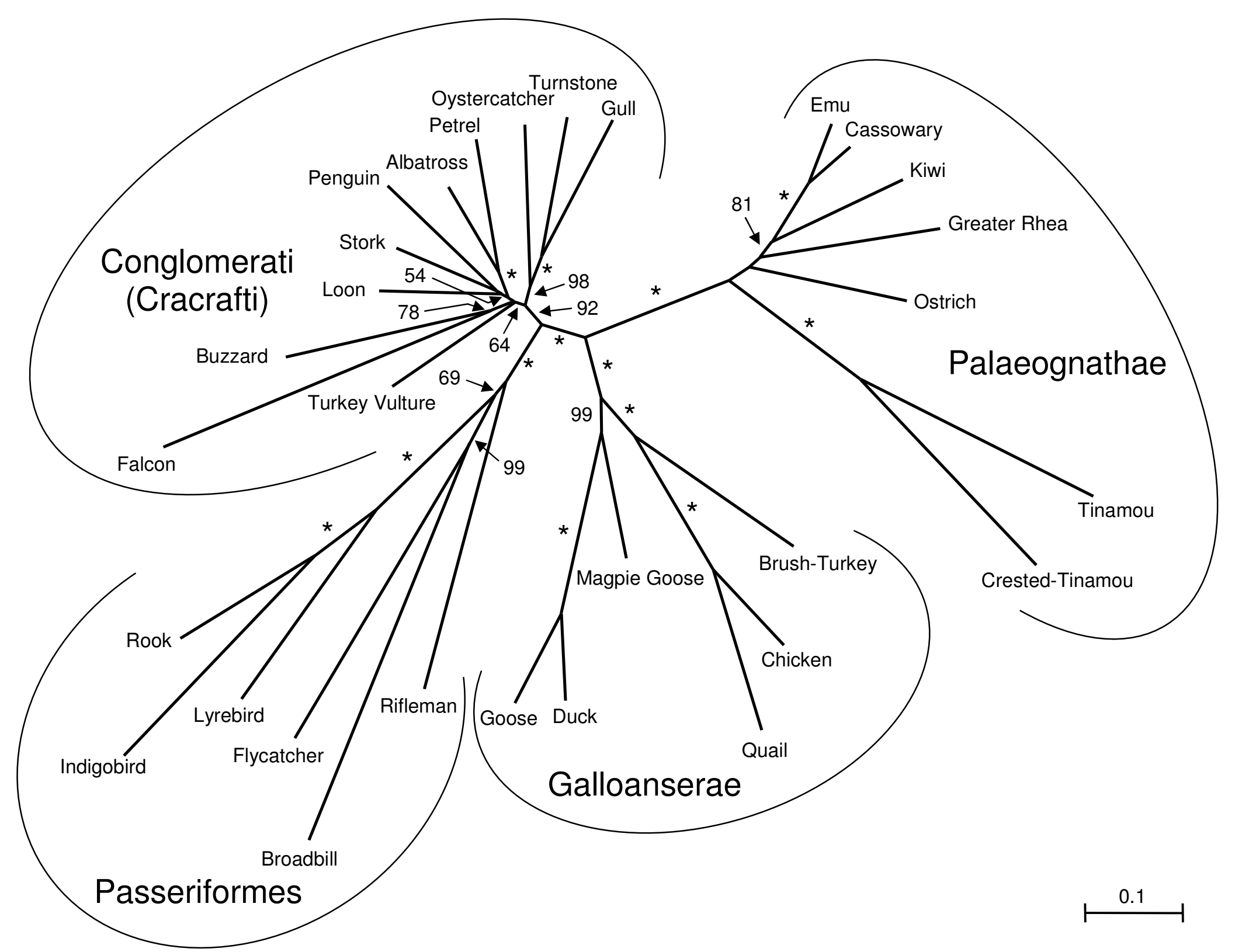

28/8/08, page 26 of 28 


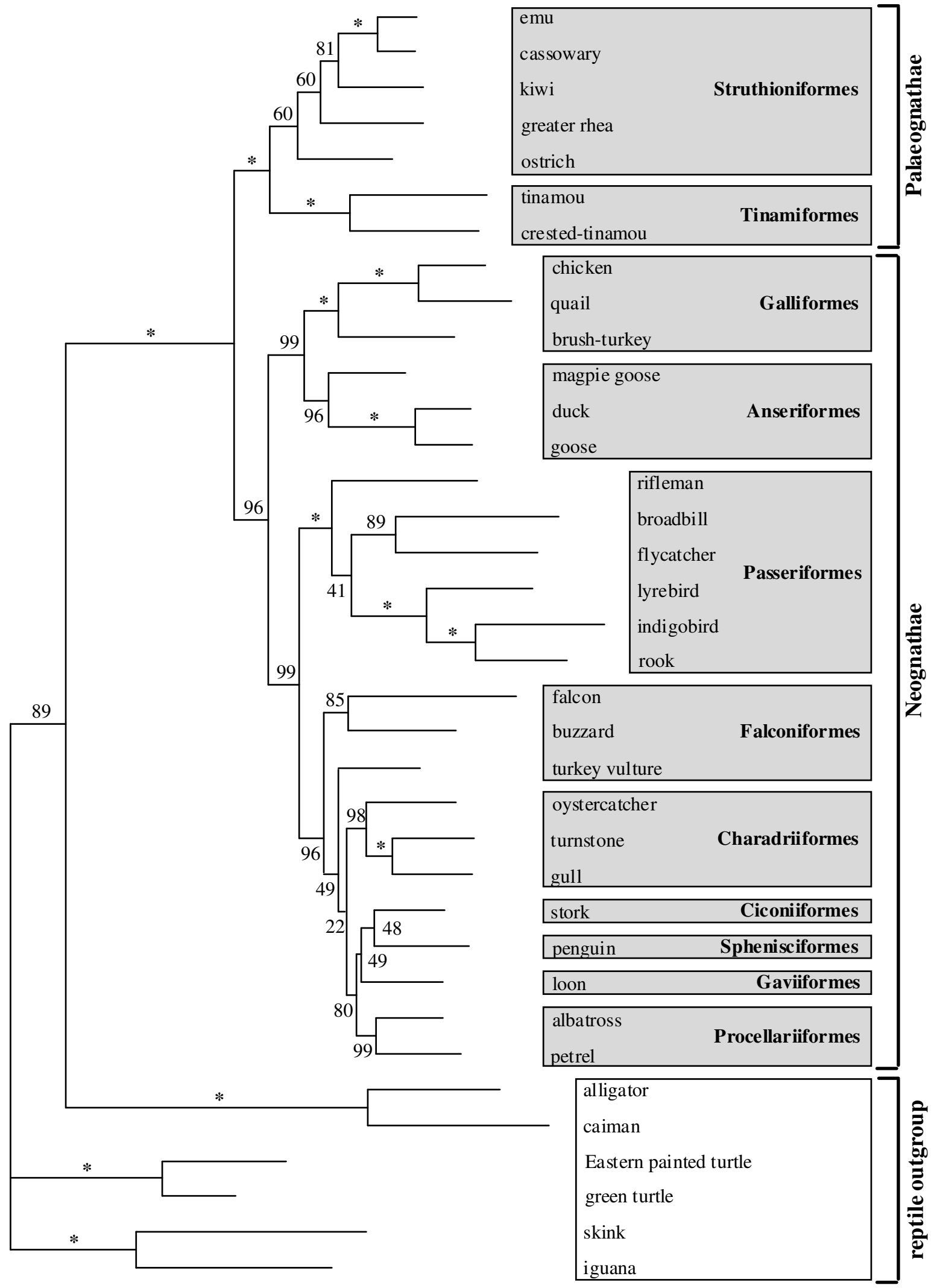

0.05 substitutions/site 


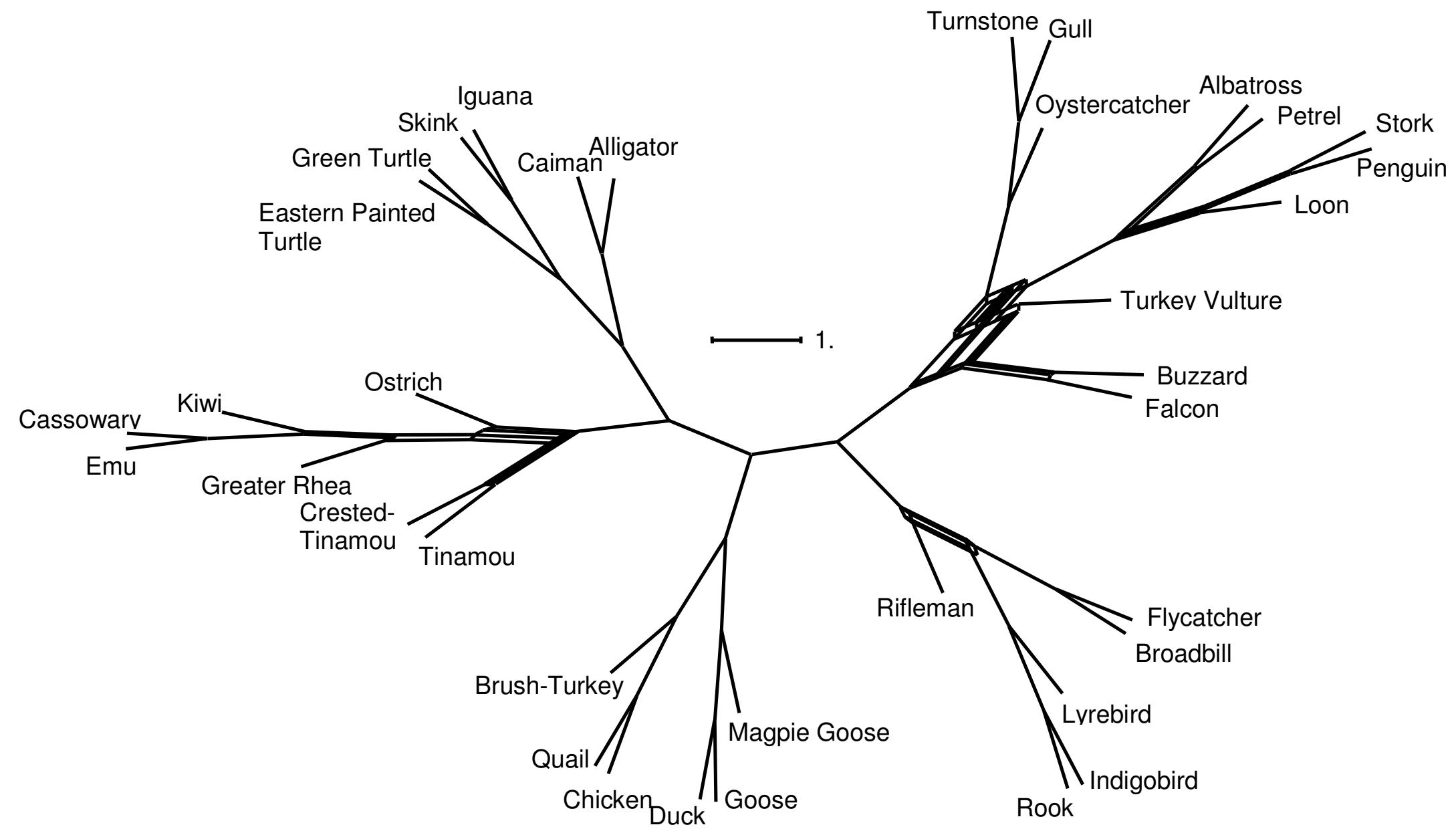

